\title{
Anemia Diagnosis on a Simple Paper-based Assay
}

\author{
Sujay K Biswas, ${ }^{1}$ Soumya Bandyopadhyay, ${ }^{2}$ Shantimoy Kar, ${ }^{3 \#}$ Nirmal K Som, ${ }^{4}$ Suman \\ Chakraborty $^{1,2,3^{*}}$
}

\begin{abstract}
1School of Medical Science and Technology, Indian Institute of Technology Kharagpur, Kharagpur, India. ${ }^{2}$ Department of Mechanical Engineering, Indian Institute of Technology Kharagpur, Kharagpur, India. ${ }^{3}$ Advanced Technology Development Centre, Indian Institute of Technology Kharagpur, Kharagpur, India. "Currently working as Postdoctoral Research Associate in Technische Universitat Darmstadt, Germany 64287, 4B C Roy Technology Hospital, Indian Institute of Technology Kharagpur, Kharagpur, India.
\end{abstract}

\begin{abstract}
In developing countries, the maternal and neonatal mortality rate is often affected by prenatal period anemia, a preventable and ubiquitous impairment attributed due to low hemoglobin (Hgb) concentration. We report the development of a simple, frugal $(\sim 0.02 \$$ per test $)$, rapid and high fidelity paper-based colorimetric microfluidic device for point-of-care (POC) detection of anemia. We validate our findings with 32 blood samples collected from different patients covering a wide spectrum of anemia and subsequently, compare with standard pathological results measured using a hematology analyzer. POC based $\mathrm{Hgb}$ estimates are correlated with the pathological gold standard estimates of Hgb levels $(r=0.909)$, and the POC test method yielded similar sensitivity and specificity for detecting mild anemia $(\mathrm{n}=8)(<11 \mathrm{~g} / \mathrm{dl})($ sensitivity: 87.5 $\%$, specificity: $100 \%)$ and for severe anemia $(\mathrm{n}=3)(<7 \mathrm{~g} / \mathrm{dl})$ (sensitivity: $100 \%$, specificity: $100 \%$ ). The estimated Hgb levels are, within $1.5 \mathrm{~g} / \mathrm{dl}$ from the pathological estimate, for $91 \%$ of the blood samples. Results demonstrate the elevated efficacy and viability of this POC colorimetric diagnostic test, in comparison to the state-of-the-art complex and expensive diagnostic tests for anemia detection.
\end{abstract}




\section{Introduction}

Anemia, a type of blood disorder, is perceived as one of the most prevalent diseases which has severely affected one-third of the world's population (2.36 billion people) $(1,2)$. The widespread causes behind anemia are fundamental hematologic ailments (e.g. sickle-cell disease, thalassemia), iron deficiency, dietary deficiencies, and chronic conditions (e.g. HIV, kidney disease, cirrhosis, inflammatory immune disorders) (3-9). Anemic patients not only experience fatigue, dizziness, and severe dehydration, but also suffer from critical complications like angina, congestive heart failure, heart diseases, and stroke(10). Anemia can trigger abated work capacity, retarded cognitive and physical growth, undesirable preterm delivery, underweight babies and thus augmented mortality risk. According to WHO, the lower threshold of hemoglobin for anemia to be $13 \mathrm{~g} / \mathrm{dl}$ for men, $12 \mathrm{~g} / \mathrm{dl}$ for non-pregnant women, and $11.5 \mathrm{~g} / \mathrm{dl}$ for children ages 5.0-11.9 years, $11 \mathrm{~g} / \mathrm{dl}$ for pregnant women and young children ages $0.5-4.99$ years (11). Anemia is diagnosed by estimating the hemoglobin level in blood, which, in turn, is dictated by the count of healthy RBCs. Early diagnosis may reduce the risk of further health complications by initiating standard treatment with necessary vitamin and dietary iron supplements. However, in the developing world, still, there is a significant dearth of rudimentary medical facilities, catering to the entire population. The paucity of clinical amenities, in conjunction with the lack of awareness, has further aggravated the current situation. Consequently, this problem attains utmost importance and entails attention from the broad scientific community.

Pathology labs $(12,13)$ ubiquitously employ cyanmethemoglobin as a standard and Drabkin's solution as the reagent for spectrophotometric quantification of hemoglobin levels. 
Kaiho and Muzino (14) expounded sensitive and rapid colorimetric assays based on 2,7diaminofluorene. Toh et al. (15) used glassy carbon electrodes coated with Nafion layers for electrochemical detection of hemoglobin by invoking the principle of cyclic voltammetry. Chitnis et al. (16) fabricated a microfluidic substrate by etching hydrophilic patterns on a hydrophobic coated paper substrate using laser treatment by $\mathrm{CO}_{2}$ laser and subsequently detected hemoglobin by adopting a luminol-based chemical pathway. Barati et al. (17) employed carbon dots as a fluorescent probe for conducting spectrofluorometric hemoglobin detection from DI water diluted blood. A multi-step procedure of scanner based hemoglobin detection method by deploying dried stain of lysed blood on chromatography paper has been reported by Yang et al (10). Taparia et al. used PDMS microchannel to detect the level of hemoglobin optically by using a CMOS sensor from diluted blood with calcium-free Tyrode buffer (18). It is undeniable that a significant progress has been made towards the development of a better methodology for point-of-care hemoglobin detection. However, none of these techniques are cost-effective and suitable for use in a resource-limited setting, still necessitate highly expensive fabrication procedure, complex detection methodology, a large volume of sample and reagent, and trained personnel. Hence, there is a need for an affordable, simple, rapid and high fidelity diagnostic platform which can be employed as a counteractive measure for the paucity of conventional pathological diagnostic platforms.

In this present work, we have demonstrated a frugal, user-friendly, and paper-based pointof-care microfluidic device for colorimetric detection of anemia. Our paper is the first report where we can reproduce gold standard results from the pathological laboratory by improvising and innovating adapted simple chemical protocols on an ultra-low-cost microfluidic chip (essentially, a piece of paper), taking cues from the fundamental chemistry governing the 
underlying colorimetric detection. In sharp contrast to other reported point of care devices for hemoglobin level detection, this device turns out to be of significant low cost, deployable with minimally trained personnel in extreme point of care settings, and capable of reproducing gold standard results from a quantitative perspective. In essence, thus, our innovative methodology not only adopted an inexpensive simple fabrication procedure with prevalent paper substrate but also enables faster and more precise quantitative detection of hemoglobin levels using minimal quantities of blood, by adopting straightforward chemical assays and uncomplicated detection algorithms, compared to existing approaches (10, 19-21). For instance, Bond et al. (22) adopted an integrated approach in quantifying hemoglobin concentrations in blood using spectrophotometer validated chip fabricated from chromatography paper. However, the delineated approach entails the involvement of expensive and cumbersome appliances and excessive quantities of blood, thereby increasing the complexity of the detection protocol. We have not only been able to surpass the accuracy of the ubiquitously used diagnostic platforms, e.g. WHO Hemoglobin Color Scale, with our proposed methodology, but also have significantly minimized the associated expenses. Furthermore, the developed frugal microfluidic platform can be easily adapted for mass production in the industry due to the straightforward manufacturing protocol, thus can be up-scaled efficiently. 


\section{Results and Discussion}

The colorimetric assay involves a Hgb catalyzed redox reaction between 3,3'-Dimethyl-[1,1'biphenyl]-4,4'-diamine (o-tolidine) and hydrogen peroxide yielding greenish-blue colored oxidized o-tolidine products (23). This reaction is mechanistically similar to the well-established and commercially adopted chemical assay which has been utilized in the spectrophotometric detection of $\mathrm{Hgb}$ in plasma, criminal inspection, and in portable POC photometers for anemia diagnosis $(19,24,25)$. We have readjusted and altered the reaction parameters (please see Supplementary Information) to obtain quantifiable colorimetric signals, corresponding to the Hgb levels spanning the entire physiological range; thereby recasting this assay into a rapid, reliable and point-of-care colorimetric detection of $\mathrm{Hgb}$ and subsequent diagnosis of anemia without any ancillary equipment.

We have explicated the kinetics of the involved chemical reaction while calibrating our paper-based microfluidic device. Reaction kinetics (Figure 2B), is depicted by the temporal variation of the normalized intensity, obtained by:

$$
I_{\text {norm }}(t)=\frac{I_{R}(t)-I_{U R}}{I_{R, M A X}-I_{U R}}
$$

(Equation 1)

The temporal variables $I_{\text {norm }}(t), I_{R}(t), I_{R, M A X}$ and $I_{U R}$ denote the normalized intensity, the intensity of the reacted pad, the average intensity of saturated color and intensity of the unreacted pad respectively. The reaction kinetics, for a particular concentration, in general, comprises three distinct phases (Figure 2A). The reaction regime (I) signify chemical reaction till its completion. The shaded regime (II) is the temporally stable saturation region; the temporal average of the intensity in this region $\left(I_{R, M A X}\right)$ has been utilized for obtaining the calibrated intensity level $(\Delta I)$. 
In the decaying regime $(I I I)$, the colorimetric signal gradually fades which impacts in the decline of the intensity of the reaction pad. Construction of the calibration curve is performed by executing repeated experiments with the serial dilutions of cyanmethemoglobin, obtained by the treatment with standard Drabkin's solution. The difference of the scaled intensities $(\boldsymbol{\Delta} I)$ is obtained for a particular trial, corresponding to a given concentration, by invoking the previously discussed formulation. The mean and the standard deviation corresponding to a set of experimental trials is obtained after filtering out the outliers outside two standard deviations from the mean and subsequently mapped with the given concentration (linear fit yields $r=0.977$ ) (Figure 3).

Our calibrated microfluidic platform is tested with human blood samples covering the entire physiologic range of Hgb. Our device has shown a significantly high degree of repeatability, corroborated by the measured intensity of the colorimetric signals, whose coefficient of variation lies below $10 \%$ (for $97 \%$ samples) (Supplementary Figure 1). The concentration of hemoglobin has been subsequently estimated from the measured intensity value, by appropriately evoking the calibration curve. Our POC anemia test obtained results, which exhibit strong correlation to those of hematology analyzers $(r=0.909)$ (Figure 4), and yielded comparable sensitivity and specificity for detecting anemia $(<11 \mathrm{~g} / \mathrm{dl})$ (sensitivity: 87.5\%, specificity: 100\%) and severe anemia (<7 g/dl) (sensitivity: 100\%, specificity: 100\%), equivalent to the state-of-the-art POC anemia diagnostic tests $(19,26-33)$. We have estimated the hemoglobin concentration, approximately within $1.5 \mathrm{~g} / \mathrm{dl}$ from the pathological estimate, for 91 $\%$ of the blood samples. The ubiquitously used WHO Hemoglobin Color Scale compares the color of a blood spot on paper with reference standards ranging from $4 \mathrm{~g} / \mathrm{dl}$ to $14 \mathrm{~g} / \mathrm{dl}$ in intervals of $2 \mathrm{~g} / \mathrm{dl}$ to quantify hemoglobin levels. In laboratory settings, the same color scale has displayed 
$95 \%$ agreement within the limits of $-3.50 \mathrm{~g} / \mathrm{dl}$ to $+3.11 \mathrm{~g} / \mathrm{dl}(33)$. This technique is susceptible to inaccuracies: Van den Broek et al. reported that the color scale yielded quantitative measurements within $2 \mathrm{~g} / \mathrm{dl}$ for only $67 \%$ of the samples (34). Our device has not only performed better in comparison to the widely used WHO Hemoglobin Color Scale Method (33, 34) in similar conditions but also has displayed an elevated degree of efficacy compared to other low-cost devices reported in the literature $(20,22)$. Results reveal the potency of our microfluidic methodology to yield a greater degree of accuracy over the existing methods with curtailed cost, in similar settings.

The combined cost of Whatman 1 filter paper, extracted to $8 \mathrm{~cm} \times 8 \mathrm{~cm}$ and combined with the requisite reagents, was estimated to have an approximately $99 \%$ reduction in expenses, relative to the HemoCue cuvettes which were priced at $\$ 0.99$ - $\$ 1.43$ per cuvette (as of January 2013), depending on the organization from which it is purchased (20). In comparison, the aggregate cost of an $8 \mathrm{~cm} \times 8 \mathrm{~cm}$ strip of the Whatman Paper and the required chromogenic reagent per test contributes to $\$ 0.0123$, which is a $99.4 \%-99.6 \%$ depreciation in terms of price from HemoCue cuvettes. The expenses incurred in printing the pattern, followed by heating the patterned paper substrate, amount to $\$ 0.0081$ per test. Hence, the aggregate cost including the material and the manufacturing cost, for the hemoglobin strip is $\$ 0.02$ (Supplementary Table 1). Considering the aggregate cost, our device is more inexpensive than the existing low-cost pointof-care devices reported in the literature $(20,33,34)$. We can reduce the expenses significantly by adopting a rapid prototyping manufacturing protocol. Our eco-friendly paper microfluidic chip, as opposed to the glass cuvettes used in pathological labs, does not necessitate intricate waste management strategies, and it can be conveniently incinerated in situ to discard detrimental biohazards. 
In conclusion, we have demonstrated a simple, affordable, rapid and high fidelity method of detecting hemoglobin from whole blood. Our paper-based platform has displayed greater sensitivity and specificity for the entire spectrum of anemic condition, relative to the prevailing methodologies; thus could potentially bridge the lacuna in the healthcare network for the resource-limited settings. 


\section{Methods}

Fabrication of the POC device: Microfluidic paper strips were fabricated on Whatman (Grade-1) cellulose filter paper [mean pore diameter $\left(2 r_{m}\right): 11 \mu \mathrm{m}, r_{m}$ stands for the mean pore radius], by ink-jet printing-based procedure, an indigenous technique developed by our group (35). Our methodology incorporates cartridge ink for creating the hydrophobic barrier surrounding the circular detection pads. The fabrication methodology is relatively simpler, frugal and suitable for large-scale manufacturing, compared to existing techniques like wax-printing $(36,37)$ and use of complex reagents for creating barriers $(38,39)$. In the adopted methodology, the prototype of the chip, to be employed, is printed on both sides of the substrate, by an ink-jet printer (HP Colour LaserJet 500). Finally, each side of the printed paper is heated to a temperature of $180^{\circ} \mathrm{C}$ for $4-5$ minutes. While heating the printed paper, toner particles melt, and molten ink diffuses through the porous paper matrix; thereby generating the hydrophobic barrier spanning across the paper thickness. Amidst the hydrophobic surface, hydrophilic reaction pads for colorimetric detection are situated, which will guide the flow of test fluid through the porous network of the paper matrix. The final fabricated prototype (Figure 1A) comprises 16 hydrophilic sites, which serve as the "reaction pads".

Color-based POC Anemia testing: We calibrated the fabricated microfluidic platform with different dilutions of cyanmethemoglobin, which were prepared by mixing varied ratios of cyanmethemoglobin standard (equivalent standard hemoglobin concentration is $15.1 \mathrm{~g} / \mathrm{dl}$ ) with Drabkin's solution. While validating the microfluidic platform, $2 \mu$ l of blood was mixed with Drabkin's solution (23RR621-80) in a volumetric ratio of 1:250, to yield cyanmethemoglobin in micro-centrifuge tubes, before being subsequently added to different hydrophilic sites of the microfluidic platform, pre-wetted with $2 \mu$ l of chromogenic reagent. The entire methodology of 
detection has been summarized in Figure 1. The extent of colorimetric reaction at the "reaction pads" was monitored by acquiring images at an interval of one second by a digital camera (Nikon D5200).

Analysis of data: The images of the hydrophilic-reaction sites were processed using the open online program ImageJ (https://imagej.nih.gov/ij/). We have chosen a time window of 150210 seconds from the reaction kinetics for selecting and processing images for our analysis. A linear transformation was judiciously employed to account for fluctuations in magnification, focusing and illumination, resulting in mapping the intensities of black $\left(I_{B R} \& I_{B U R}\right)$ and white $\left(I_{W R}\right.$ $\left.\& I_{W U R}\right)$ regions of the microfluidic device to 0 and 255 respectively. The subscripts 'R' and 'UR' denote the intensities corresponding to the reacted and unreacted reaction pads respectively. The intensity of the image of the reaction pad, wetted with the chromogenic reagent, before the onset of chemical reaction $\left(I_{U R}\right)$ was first calculated and linearly transformed. Dynamic variation of intensity of the reaction pad during the course of the chemical reaction was explicated and discussed in detail in the "Results and Discussion". The temporal average of the intensity $\left(I_{R, M A X}\right)$ in an appropriate timeframe, after the completion of the chemical reaction, was subsequently obtained and scaled accordingly. The difference of the scaled intensities $(\Delta I)$, corresponding to a given concentration, was obtained by:

$$
\frac{\Delta I}{255}=\frac{I_{R, M A X}-I_{B R}}{I_{W R}-I_{B R}}-\frac{I_{U R}-I_{B U R}}{I_{W U R}-I_{B U R}}
$$

(Equation 2)

Statistical Analysis: All statistical analyses were performed using MATLAB 2017. Pearson correlations, linear regression models, and prediction bounds were employed to estimate the correlation between Hgb levels via pathological hematology analyzer and POC device. 
Sensitivity and specificity results were computed for the entire set of patients, irrespective of the age group.

Study approval: An approval of ethical clearance was taken from Institute Ethical Committee (IEC) for the commencement of this study. Pediatric and adult patient blood samples were obtained from the pathology clinic of the B.C. Roy Technology Hospital. All specimens were collected after informed approval was received from patients or their parents/guardians. Each consented patient only provided one sample on the day of consent. 


\section{Conflicts of interest}

The authors have declared that no conflict of interest exists.

\section{Author Contributions}

SC conceptualized and envisioned this study. SKB and SB were responsible for the design, fabrication, experiments, post-processing, and analysis. SK was involved in technical design and strategy for execution of the study with SKB and SB. NKS actively involved in the clinical validation part of the work. All members were involved in technical discussion in different stages of the project and contributed to manuscript writing.

\section{Acknowledgements}

This work is a part of the project entitled "Development of Smartphone Integrated Generic Microfluidic Devices for Rapid, Portable and Affordable Point-of-Care Diagnostics" (Project ID: 4429) (GDD), funded by a grant from the Ministry of Human Resource Development (MHRD) and Indian Council of Medical Research (ICMR), Department of Health Research, Ministry of Health and Family Welfare, New Delhi. This material is based upon work supported by the MHRD Research Fellowship from Government of India, MHRD, Department of Higher Education, New Delhi. We appreciate the collaborative support from BC Roy Technology Hospital, Indian Institute of Technology for providing blood samples and their pathological test results. 


\section{References}

1. Vos $\mathrm{T}$ et al. Global, regional, and national incidence, prevalence, and years lived with disability for 310 diseases and injuries, 1990-2015: a systematic analysis for the Global Burden of Disease Study 2015. Lancet 2016;388(10053):1545-1602.

2. World Health Organization 2015. The Global Prevalence of Anaemia in 2011. WHO Rep. $2011 ; 48$.

3. Calabrich A, Katz A. Management of anemia in cancer patients. Futur. Oncol. 2011;7(4):507517.

4. Koukounari A et al. Relationships between anaemia and parasitic infections in Kenyan schoolchildren: A Bayesian hierarchical modelling approach. Int. J. Parasitol. 2008;38(14):1663-1671.

5. Bechir M, Schelling E, Hamit MA, Tanner M, Zinsstag J. Parasitic infections, anemia and malnutrition among rural settled and mobile pastoralist mothers and their children in chad. Ecohealth 2012;9(2):122-131.

6. Beghé C, Wilson A, Ershler WB. Prevalence and outcomes of anemia in geriatrics: A systematic review of the literature. Am. J. Med. 2004;116(7 SUPPL. 1):3-10.

7. Powars DR, Schroeder WA, Weiss JN, Chan LS, Azen SP. Lack of influence of fetal hemoglobin levels or erythrocyte indices on the severity of sickle cell anemia.. J. Clin. Invest. 1980;65(3):732-740.

8. Noguchi CT et al. Alpha thalassemia changes erythrocyte heterogeneity in sickle cell disease..

J. Clin. Invest. 1985;75(5):1632-1637.

9. Bateman JC, Shorr HM, Elgvin T. HYPERVOLEMIC ANEMIA IN CIRRHOSIS. J. Clin. Invest. 1949;28(3):539-547.

10. Yang $X$ et al. Simple paper-based test for measuring blood hemoglobin concentration in resource-limited settings. Clin. Chem. 2013;59(10):1506-1513.

11. McLean E, Cogswell M, Egli I, Wojdyla D, De Benoist B. Worldwide prevalence of anaemia, WHO Vitamin and Mineral Nutrition Information System, 1993-2005. Public Health Nutr. 2009;12(4):444-454.

12. Eilers RJ. Notification of final adoption of an international method and standard solution for hemoglobinometry specifications for preparation of standard solution. Am. J. Clin. Pathol. 1967;47(2):212-214. 
13. Van Kampen EJ, Zijlstra WG. Standardization of hemoglobinometry II. The hemiglobincyanide method. Clin. Chim. Acta 1961;6(4):538-544.

14. Kaiho S ichi, Mizuno K. Sensitive assay systems for detection of hemoglobin with 2,7diaminofluorene: Histochemistry and colorimetry for erythrodifferentiation. Anal. Biochem. 1985;149(1):117-120.

15. Toh RJ, Peng WK, Han J, Pumera M. Direct in vivo electrochemical detection of haemoglobin in red blood cells. Sci. Rep. 2014;4:1-6.

16. Chitnis G, Ding Z, Chang C-L, Savran CA, Ziaie B. Laser-treated hydrophobic paper: an inexpensive microfluidic platform. Lab Chip 2011;11(6):1161.

17. Barati A, Shamsipur M, Abdollahi H. Hemoglobin detection using carbon dots as a fluorescence probe. Biosens. Bioelectron. 2015;71:470-475.

18. Taparia N, Platten K, Anderson K, Sniadecki N. A microfluidic approach for hemoglobin detection in whole blood. AIP Adv. 2017;7:105102-11.

19. Tyburski EA et al. Disposable platform provides visual and color-based point-of-care anemia self-testing. J. Clin. Invest. 2014;124(10):4387-4394.

20. Gómez-Simón A et al. Evaluation of four rapid methods for hemoglobin screening of whole blood donors in mobile collection settings. Transfus. Apher. Sci. 2007;36(3):235-242.

21. Larson DL, Ranney HM. FILTER PAPER ELECTROPHORESIS OF HUMAN HEMOGLOBIN. J. Clin. Invest. 1953;32(11):1070-1076.

22. Bond $\mathrm{M}$ et al. Chromatography paper as a low-cost medium for accurate spectrophotometric assessment of blood hemoglobin concentration. Lab Chip 2013;13(12):2381-2388.

23. Goyal MM, Basak A. Estimation of plasma haemoglobin by a modified kinetic method using o-tolidine. Indian J. Clin. Biochem. 2009;24(1):36-41.

24. McMurdy JW, Jay GD, Suner S, Crawford G. Noninvasive optical, electrical, and acoustic methods of total hemoglobin determination. Clin. Chem. 2008;54(2):264-272.

25. Laberke P, Hausmann R, Wiprachtiger N, Briellmann T, Balitzki B. Analysis with the Combur-Test — special aspects in forensic trace examination. Arch. Kriminol. 2012;229(56):189-197.

26. Lamhaut $\mathrm{L}$ et al. Comparison of the accuracy of noninvasive hemoglobin monitoring by spectrophotometry $(\mathrm{SpHb})$ and hemocue ${ }^{\circledR}$ with automated laboratory hemoglobin measurement. Anesthesiology 2011;115(3):548-554. 
27. Critchley J, Bates I. Haemoglobin colour scale for anaemia diagnosis where there is no laboratory: a systematic review. Int. J. Epidemiol. 2005;34(6):1425-1434.

28. Ingram CF, Lewis SM. Clinical use of WHO haemoglobin colour scale: Validation and critique. J. Clin. Pathol. 2000;53(12):933-937.

29. White SA, van den Broek NR. Methods for assessing reliability and validity for a measurement tool: A case study and critique using the WHO haemoglobin colour scale. Stat. Med. 2004;23(10):1603-1619.

30. Dewhirst E et al. Accuracy of Noninvasive and Continuous Hemoglobin Measurement by Pulse Co-Oximetry During Preoperative Phlebotomy. J. Intensive Care Med. 2014;29(4):238242.

31. Aldridge C, Foster HME, Albonico M, Ame SM, Montresor A. Evaluation of the diagnostic accuracy of the Haemoglobin Colour Scale to detect anaemia in young children attending primary healthcare clinics in Zanzibar. Trop. Med. Int. Heal. 2012;17(4):423-429.

32. Nkrumah B et al. Hemoglobin estimation by the HemoCue ${ }^{\circledR}$ portable hemoglobin photometer in a resource poor setting. BMC Clin. Pathol. 2011;11(1):5.

33. Paddle JJ. Evaluation of the Haemoglobin colour scale and comparison with the HemoCue haemoglobin assay. Bull. World Health Organ. 2002;80(10):813-816.

34. Van Den Broek NR, Ntonya C, Mhango E, White SA. Diagnosing anaemia in pregnancy in rural clinics: Assessing the potential of the haemoglobin colour scale. Bull. World Health Organ. 1999;77(1):15-21.

35. Dey R, Kar S, Joshi S, Maiti TK, Chakraborty S. Ultra-low-cost 'paper-and-pencil' device for electrically controlled micromixing of analytes. Microfluid. Nanofluidics 2015;19(2):375383.

36. Lu Y, Shi W, Qin J, Lin B. Fabrication and characterization of paper-based microfluidics prepared in nitrocellulose membrane by Wax printing. Anal. Chem. 2010;82(1):329-335.

37. Carrilho E, Martinez AW, Whitesides GM. Understanding Wax Printing: A Simple Micropatterning Process for Paper-Based Microfluidics. Anal. Chem. 2009;81(16):7091-7095.

38. Matsuura K et al. Paper-based diagnostic devices for evaluating the quality of human sperm. Microfluid. Nanofluidics 2014;16(5):857-867.

39. Chen $\mathrm{C}$ et al. Paper-based immunoaffinity devices for accessible isolation and characterization of extracellular vesicles. Microfluid. Nanofluidics 2014;16(5):849-856. 

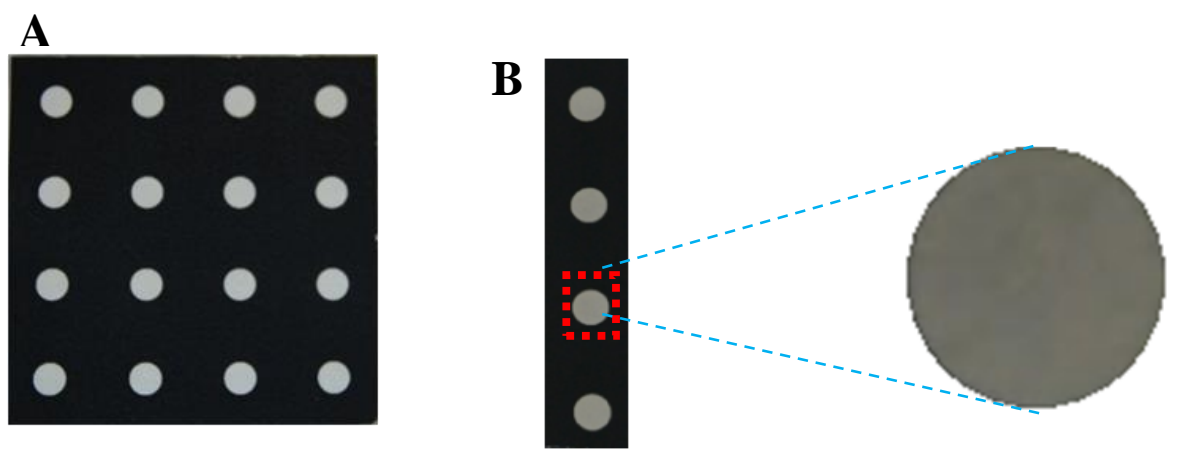

C

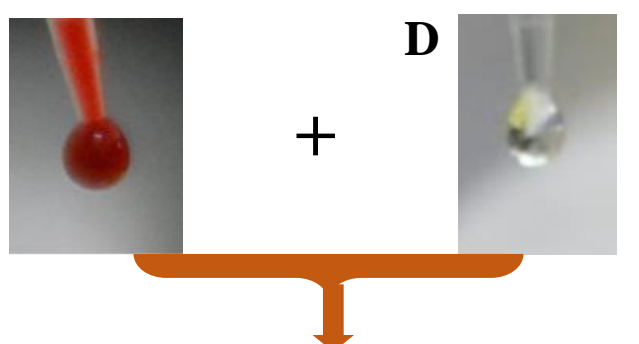

$\mathbf{E}$
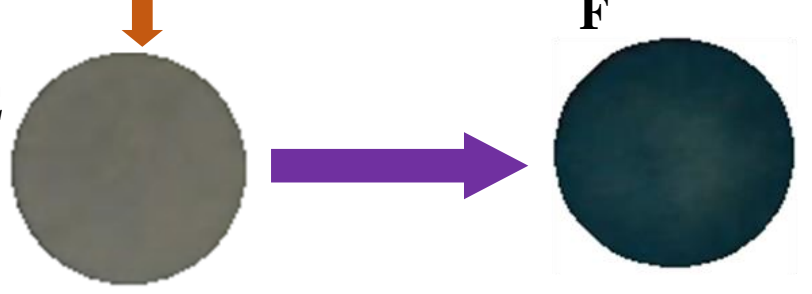

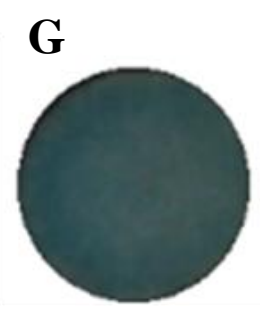

$2.5 \mathrm{~g} / \mathrm{dl}$

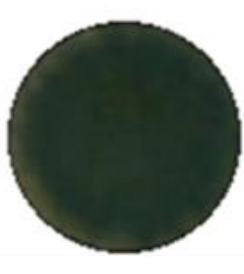

$5 \mathrm{~g} / \mathrm{dl}$

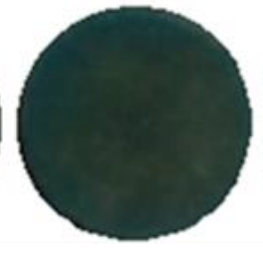

$7.5 \mathrm{~g} / \mathrm{dl}$

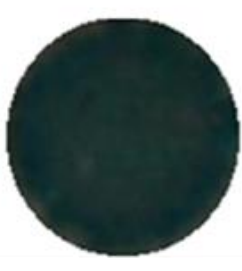

$10.0 \mathrm{~g} / \mathrm{dl}$

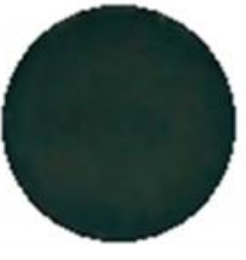

$12.5 \mathrm{~g} / \mathrm{dl}$

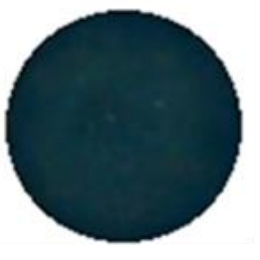

$15.1 \mathrm{~g} / \mathrm{dl}$

Figure 1. The POC color-based anemia test. (A) The microfluidic POC chip comprises 16 circular detection sites of diameter $0.7 \mathrm{~cm}$ each, separated by a hydrophobic barrier, in a square array $(8 \mathrm{~cm} \times 8 \mathrm{~cm})$. (B-F) Steps for using the POC color-based anemia test. (B) Depicts the magnified view of a reagent immobilized reaction site. (C) Blood, mixed with Drabkin's solution (D) added to the detection sites (E). (F) Wait until complete coloration and interpret developed color of the detection site. (G) Ranging from lighter shade of bluish green to deeper shade, resultant solution colors map with different Hgb levels, as outlined. 

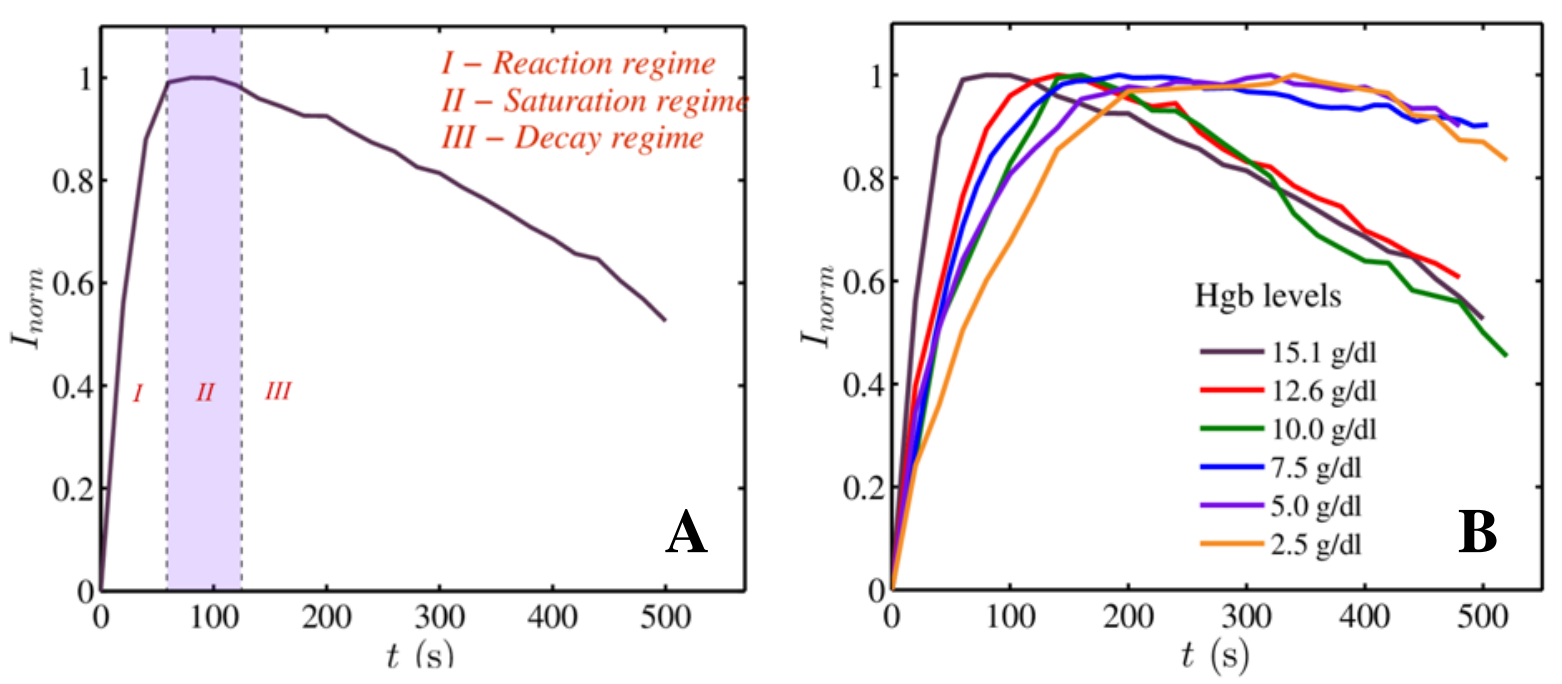

Figure 2. Quantification of the reaction kinetics of POC device for color-based anemia test. (A) Plot depicting the variation of normalized intensity $\left(I_{\text {norm }}\right)$ with time $(t)$. Different regimes of colorimetric reaction are outlined. Normalized intensity maxima lie in the regime II. (B) Curves depicting reaction kinetics of resultant cyanmethemoglobin solution for blood samples with Hgb levels of $2.5 \mathrm{~g} / \mathrm{dl}, 5.0 \mathrm{~g} / \mathrm{dl}, 7.5 \mathrm{~g} / \mathrm{dl}, 10.0 \mathrm{~g} / \mathrm{dl}, 12.6 \mathrm{~g} / \mathrm{dl}$, and $15.1 \mathrm{~g} / \mathrm{dl}$.

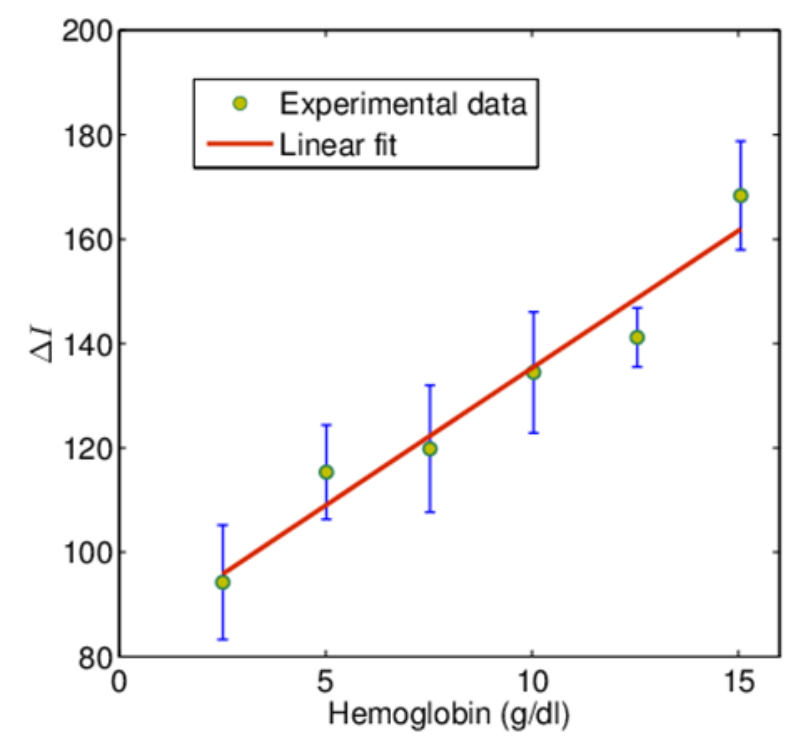

Figure 3. Standard calibration curve of POC device for color-based anemia test. A standard curve was constructed using serial dilutions of standard hemoglobin to create $\mathrm{Hgb}$ levels ranging between $2.5 \mathrm{~g} / \mathrm{dl}$ and $15.1 \mathrm{~g} / \mathrm{dl}$. $R$ value is 0.977 . The error bars in the plot represent the standard deviation in the mean intensity computed from 20 trials corresponding to a given dilution. 


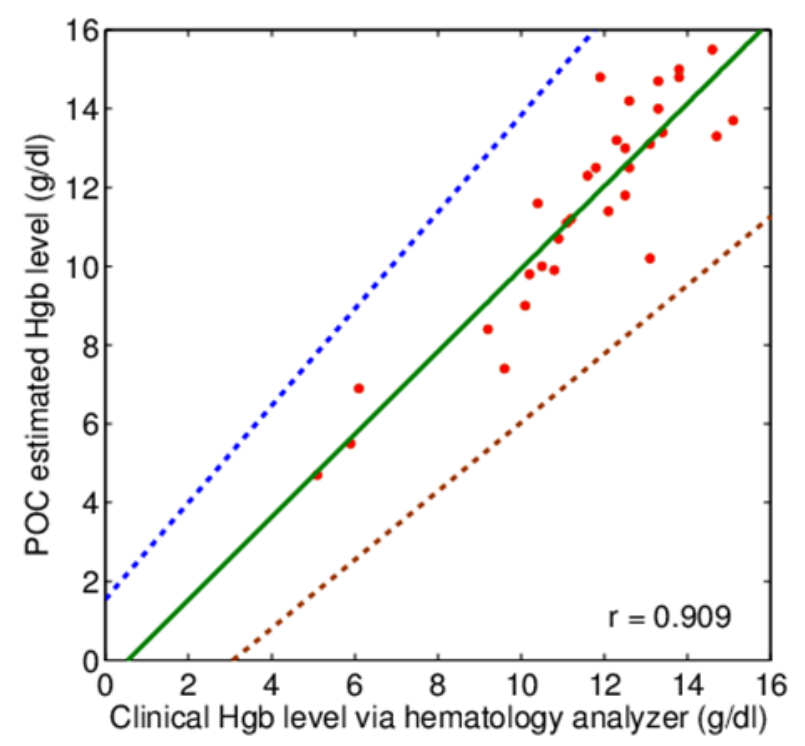

Figure 4. Clinical assessment of POC colorimetric anemia test results. POC Hgb levels are plotted against clinical Hgb levels determined via a hematology analyzer for all patient samples $(n=32)$, depicting a strong correlation $(r=0.909)$. Furthermore, 95\% prediction limits for the individual values were computed (dashed lines). 